\title{
A Study of the Molecular Mechanism by Which Substance P Enhances Doxorubicin (Adriamycin)- Associated Autophagy and Apoptosis of Cardiomyocytes Induces Heart Failure
}

Fa-Xiu CHEN CHEN ( $\square$ mahmoud.abuharb@yahoo.com )

Dalian Medical University https://orcid.org/0000-0003-2337-872X

Jian $\mathrm{Hu}$

Jiangxi Provincial People's Hospital

Jing Fang

Jiangxi Provincial People's Hospital

Qing-Ling Li

Jiangxi Provincial People's Hospital

Qin Wan

Jiangxi Provincial People's Hospital

Le Peng

Jiangxi Provincial People's Hospital

Research article

Keywords: Substance P, Doxorubicin, Heart failure, Apoptosis, Autophagy, Molecular

Posted Date: June 30th, 2021

DOl: https://doi.org/10.21203/rs.3.rs-659015/v1

License: (c) This work is licensed under a Creative Commons Attribution 4.0 International License.

Read Full License 


\section{Abstract}

Background: Anthracyclines, including doxorubicin, are some of the most potent anticancer drugs available. However, the use of doxorubicin as a chemotherapeutic agent is severely hindered by doselimiting toxicity, particularly cardiotoxicity, while degrading other organ systems. Despite years of use and the amount of details published on this drug, the understanding of its cellular mechanisms is still incomplete.

Method: Cardiomyocyte grouping was carried out, where H9C2 cardiomyocytes were randomly divided into a control group, a myocardial model group, an SP group and an SP antagonist group. For replication in animal models, twelve rats were similarly divided randomly into a control group, a myocardial model group, an SP group, and an SP antagonist group. Except for the control group, the rats in the other groups were modelled: the rats were injected with adriamycin solution. Flow cytometry was used to detect apoptosis, while HE staining, TUNEL stain, Western Blot detection and transmission electron microscopy were performed to detect autophagy levels accordingly. All the results were analysed and carefully interpreted.

Results: The tachykinin, substance $\mathrm{P}$, is located mainly in sensory nerves and in the heart, where substance P-containing nerve fibres are often found around coronary vessels, making them ideally situated to detect changes in the myocardial environment. Apoptosis and autophagy are geneticallyregulated, evolutionary-conserved processes that regulate cell fate and are both important for development, normal physiology, and a wide range of diseases. Recent studies show that despite the significant differences between these two processes, their regulation is closely connected and certain regulators have been found to be able to control both apoptosis and autophagy.

Conclusions: In this study, the influence of substance $\mathrm{P}$ was discussed, providing possible molecular mechanisms for crosstalk between apoptosis and autophagy mediating heart failure due to Doxorubicin therapy.

Trial registration: (20192BBGL7D031) 2019-09-17.

\section{Background}

In physiological conditions, autophagy and apoptosis play essential roles in cardiac health and integrity, with the structure and function of cardiac myocytes closely related to autophagic flux. Cardiac myocytes retain a limited ability to re-enter the cell cycle, leading to a limited regeneration capacity in the adult heart. As a result, a continuous process of cell renovation including the removal and replacement of damaged tissue is present. In addition, autophagy is necessary for continual heart contraction and large cytoplasmic calcium transients without disrupting cardiac function [1]. During heart development, apoptosis is involved in the development of the embryonic outflow tract, cardiac valves, conducting system, and coronary vasculature [2]. In pathological conditions, the interplay between autophagy and 
apoptosis is closely linked to certain cardiac diseases including ischaemic heart disease, pressure overload-induced cardiac disease, and diabetic cardiomyopathy.

Substance $\mathrm{P}(\mathrm{SP})$ belongs to the tachykinin family of sensory nerve neuropeptides and other classical members of this family include neurokinin A (NKA) and neurokinin B (NKB). Although NKB is encoded by its gene (TAC2) and is restricted to the central nervous system, SP and NKA are both encoded by the TAC1 gene and are located in the central nervous system and peripheral afferent sensory neurons [3].

There has been focus on SP due to its good and bad sides in myocardial remodelling and heart failure. It offers protection following ischaemia-reperfusion [4], however, it also causes long-term non-ischaemic myocardial remodelling and heart failure [5].

Although there are obvious morphological differences between autophagy and apoptosis, they are actually functionally related. In recent years, studies have discovered multiple interactions between apoptosis and autophagy, namely crosstalk [6], which occurs mainly through the following 3 pathways: (1) p53 pathway: When the body encounters ischaemia, hypoxia, DNA damage, and other stress, the p53 gene is activated, playing a dual regulatory role by affecting both cell apoptosis and autophagy; (2) Beclin1 pathway: Autophagy regulatory factors can be combined with different Beclin1 domains (ECD, $\mathrm{CCD}, \mathrm{BH} 3$ ) to achieve either positive or negative regulation of autophagy levels, while the anti-apoptotic proteins $\mathrm{Bcl}-2$ and $\mathrm{Bcl}-\mathrm{XL}$ can bind to the $\mathrm{BH} 3$ domain of Beclin1, inhibiting its anti-apoptotic effect and autophagy; (3) BH3-only pathway: The BH3-only protein is a pro-apoptotic protein that either directly activates the $\mathrm{BH} 123$ protein or antagonises the $\mathrm{BH} 1234$ protein. It can also interfere with the binding of Bcl-2 protein and Beclin-1 protein to release free Beclin-1 and activate autophagy [7-9] (see Figure 1). Studies have found that treating rats with heart failure using drugs can effectively reduce cardiomyocyte autophagy and inhibit cardiomyocyte apoptosis, thereby improving the heart function of the rats [10]. Although apoptosis and autophagy systems can perform their functions independently, they are also clearly related to each other with both systems jointly regulating cell biological processes through crosstalk, thus participating in the occurrence and development of heart failure. It is currently believed that apoptosis-autophagy crosstalk can provide new ideas and directions for the study of heart failure pathogenesis and therapeutic targets.

Substance $\mathrm{P}(\mathrm{SP})$ is released in large quantities when the heart is noxiously stimulated, and plays a dual role in the occurrence and development of heart failure: During the acute ischaemic phase of the heart, SP exerts myocardial protection through strong vasodilation, especially in the diastolic coronary arteries. Both non-ischaemic-induced myocardial remodelling and chronic heart failure are detrimental [12]. Several studies, including Valdemarsson [13], confirmed that the expression of SP in the plasma of patients with chronic heart failure is significantly higher than that of healthy people. Moreover, the SP level in plasma of patients with heart failure and angina symptoms is higher than patients without angina [14].

The studies mentioned above suggested that SP plays a pathogenic role in the pathogenesis of heart failure. However, the molecular mechanism of SP-mediated heart failure is not yet clear. In recent years, 
studies have shown that the increase of SP expression whether in hair tissue or bladder tissue significantly improves autophagy activity $[15,16]$ and that SP has a clear induction effect on autophagy. In this study, doxorubicin was used to induce cardiomyocyte damage in vitro and exogenous SP was used for intervention during the preliminary test. Findings showed that the expression of cardiomyocyte autophagy marker Beclin-1 significantly and the apoptosis rate of cardiomyocytes significantly increased. These preliminary experimental results indicated that the mechanism of SP-mediated heart failure could be related to the regulation of autophagy/apoptosis [17], but remained unclear.

\section{Methods}

The study was submitted to and approved by the Key Research and Development Programme of Jiangxi Province ethics committee.

\section{Experimental animal}

Twelve male nude mice were purchased from Hunan Slack Jingda Experimental Animal Co., Ltd., licence number: SCXK (Xiang) 2019-0004).

\section{Experimental cell}

H9C2 cardiomyocytes were purchased from Beijing Beina Chuanglian Biotechnology Research Institute, batch number: BNCC295057.

\section{Main reagents and instruments}

Complete Dulbecco's Modified Eagle Medium (DMEM) medium (KGM12800S-500, KGI Bio), trypsinEthylenediaminetetraacetic acid (EDTA) digestion solution (T1300, Solarbio), Phosphate-buffered saline (PBS) (KGB5001, KGI Bio), hematoxylin staining solution (ZLI-9610, Zhongshan Jinqiao), eosin Staining solution (G1100, Solarbio), ultra-clean advanced mounting glue (YZB, BASO), Scott blue solution (G1865,

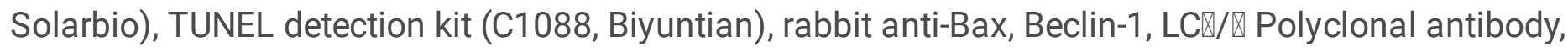
mouse anti-Bcl-2 antibody (A0207, ab62557, bs-8878R, ab692, American Abcam), horseradish enzyme labelled goat anti-rabbit IgG (H+L) (ZB-2301, Beijing Zhongshan Jinqiao Biotechnology Co., Ltd.

Technology Co., Ltd.), DAB colour reagent kit (CW0125, CWBIO), neutral resin (CW0136, CWBIO), Annex VFITC/PI Apoptosis Kit (AP101-100-kit, MULTI SCIENCES), Amylum Hydrochloride Su (Shenzhen Wanle Pharmaceutical Co., Ltd., batch number: 1812E1), SP (MCE, lot number: 25994), Aprepitant (MCE, lot number: 31982). CO2 incubator (BPN-80CW, Shanghai Yiheng Scientific Instrument Co., Ltd.), multifunctional microplate reader (WD-2102B, Beijing Liuyi Biotechnology Co., Ltd.), fluorescent PCR machine (CFX Connect ${ }^{\text {TM }}$ real-time, Bole Life Medical Products ( Shanghai) Co., Ltd.), microtome (BQ318D, Bernard), fluorescence microscope (CKX53, OLYMPUS), inverted fluorescence microscope (MF53, Guangzhou Mingmei Optoelectronics Co., Ltd.), electrothermal blast drying oven (DHG-9070A, constant Scientific Instruments Co., Ltd.), NovoCyte ${ }^{\text {TM }}$ flow cytometer (NovoCyte 2060R, ACEM (Hangzhou) Co., Ltd.). 


\section{Cardiomyocyte grouping}

H9C2 cardiomyocytes were randomly divided into a control group, a myocardial model group $(2 \mu \mathrm{m}$ doxorubicin induction), an SP group ( $2 \mu \mathrm{m}$ doxorubicin $+1 \mu \mathrm{g} / \mathrm{ml}$ exogenous SP), and an SP antagonist group ( $2 \mu \mathrm{m}$ doxorubicin induction) Doxorubicin $+5 \mu \mathrm{M} \mathrm{SP}$ antagonist).

\section{Flow cytometry to detect apoptosis}

$1 \times 10^{6}-3 \times 10^{6}$ cells were collected, then added with $1 \mathrm{ml} \mathrm{PBS}$, centrifuged at $1500 \mathrm{rpm}$ for 3 minutes, and washed twice. A $5 \times$ Binding Buffer was diluted to $1 \times$ with double distilled water and 300 ul of prechilled $1 \times$ Binding Buffer was used to resuspend the cells. 3 ul Annexin V-FITC and 5 ul PI-PE were added to each tube. After mixing slightly, each tube was incubated for 10 minutes at room temperature in the dark, then 200 ul of pre-cooled $1 \times$ Binding Buffer was added to each tube. After mixing, the mixtures were tested using a flow meter.

\section{Animal model replication and drug treatment}

Twelve rats were randomly divided into a control group, a myocardial model group, an SP group, and an SP antagonist group. Except for the control group, the rats in the other groups were modelled: the rats were injected with the prepared adriamycin solution at a dose of $3 \mathrm{mg} / \mathrm{kg}$ (the adriamycin concentration was $1 \mathrm{mg} / \mathrm{ml}$, so the injection volume was $3 \mathrm{ml} / \mathrm{kg}$ ). The injection was done once every 3 days, 5 times in a row. In the same period, the control group was intraperitoneally injected with the same amount $(3 \mathrm{ml} / \mathrm{kg})$ of normal saline once every 3 days for 5 consecutive times. Every 4 days, the SP group was injected with exogenous SP through the tail vein at a dose of $6.7 \mu \mathrm{g} / \mathrm{kg}$ (exogenous SP concentration was $1 \mu \mathrm{g} / \mathrm{ml}$, so the injection volume was $6.7 \mathrm{ml} / \mathrm{kg}$ ), the SP antagonist group injected with the SP antagonist at a dose of $6.7 \mu \mathrm{g} / \mathrm{kg}$ (SP antagonist concentration was $1 \mu \mathrm{g} / \mathrm{ml}$, so the injection volume was $6.7 \mathrm{ml} / \mathrm{kg}$ ), and both the control and myocardial model group were injected with the same amount $(6.7 \mathrm{ml} / \mathrm{kg})$ normal saline.

\section{HE staining}

The tissues were taken out and rinsed with running water for several hours, dehydrated by $70 \%, 80 \%$, and $90 \%$ ethanol solutions, mixed with pure alcohol and xylene for 15 minutes, xylene I for 15 minutes, and xylene II for 15 minutes (until transparent). Then, the tissues were placed in a mixture of xylene and paraffin in half for 15 minutes, followed by placement in paraffin I and paraffin II for 50-60 minutes each before embedded in paraffin and sectioned. The paraffin slices were baked, dewaxed, and hydrated then put in a hematoxylin aqueous solution, with distilled water added, and stained for 3 minutes. Subsequently, the slices were placed into a hydrochloric acid ethanol differentiation solution for 15 seconds and washed with water. The solution was allowed to turn back to blue for 15 seconds then rinsed with running water and stained with eosin for 3 minutes. Finally, the samples were rinsed with running water, dehydrated, and covered with a transparent film for microscopic examination.

\section{TUNEL stain}


The tissue sections were placed in xylene for 10 minutes. After that, xylene was removed and the samples were allowed to deparaffinise for 10 minutes, before being put in gradient ethanol for hydration. The slices were then placed into a wet box, with $50 \mu \mathrm{g} / \mathrm{ml}$ Proteinase $\mathrm{K}$ working solution added dropwise to each sample, and allowed to react at $37^{\circ} \mathrm{C}$ for 30 minutes for antigen and antibody retrieval. Following that, the slices were washed thoroughly with PBS 3 times for 5 minutes each time. An absorbent paper was used to absorb the PBS around the tissue, with a sufficient amount of TUNEL detection solution added to each slide, and the samples were incubated for 2 hours at $45^{\circ} \mathrm{C}$ in the dark. Then, the samples were washed with PBS 3 times for 5 minutes each time and the liquid was blotted on a glass slide with absorbent paper. Finally, the slides were mounted with anti-fluorescence quenching and observed under a fluorescence microscope.

\section{Western Blot detection}

The tissue was taken and ground into a powder with liquid nitrogen, then the lysis solution was added and the tissue was ground again. Following this, the tissue homogenate was sucked into an Eppendorf (EP) tube, lysed on ice for 30 minutes, centrifuged at 12,000 rpm for 15 minutes and the supernatant was carefully aspirated to obtain the total protein. The protein concentration was determined according to the BCA kit, then the protein was denatured, loaded, and subjected to sodium dodecylbenzene sulfonate gel electrophoresis (SDS-PAGE) for 2 hours. It was then transferred to the membrane with a constant current of $300 \mathrm{~mA}$ for 80 minutes. The primary antibody solution was incubated overnight at $4{ }^{\circ} \mathrm{C}$ before incubation with the secondary antibody solution for 2 hours at room temperature. The Chemiluminescence (ECL) luminescent liquid was dropped onto the film and exposed in the gel imaging system. The "Quantityone" software was used to analyse the grey value of each antibody band.

\section{Transmission electron microscopy to detect autophagy levels}

After the embedded paraffin sections were deparaffinised and dehydrated, the sections were cut into 80 $\mathrm{nm}$ thickness using an ultra-thin microtome, and then stained with $3 \%$ uranyl acetate-lead citrate, washed with distilled water, and the autophagosomes were observed under a transmission electron microscope.

\section{Results}

\section{The effect of SP on the viability of $\mathrm{H} 9 \mathrm{C} 2$ cells induced by Adriamycin}

As shown in Figure 2, the cell proliferation rate of the myocardial model group was significantly reduced compared to the control group $(P<0.05)$ while a comparison between the myocardial model and SP group showed that there was a significant increase in the cell proliferation rate of the SP group $(P<0.05)$. For the SP antagonist group, there was a significantly decreased cell proliferation rate compared to the SP group $(P<0.05)$.

\section{The effect of SP on $\mathrm{H} 9 \mathrm{C} 2$ cell apoptosis induced by Adriamycin}


Figure 3 demonstrates that the apoptosis rate of the myocardial model group was significantly increased compared to the control group $(P<0.05)$, while the apoptosis rate of the SP group was significantly decreased compared to the myocardial model group $(P<0.05)$, and the SP antagonist group mortality rate was significantly increased compared to the SP group $(P<0.05)$.

\section{The effect of SP on Bax, Bcl-2, Beclin-1, LCD/ $\square$ protein expressions in $\mathrm{H} 9 \mathrm{C} 2$ cells induced by Adriamycin}

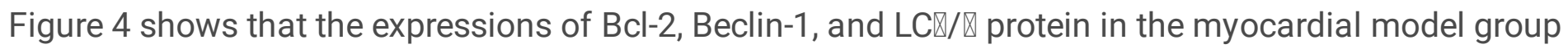
were significantly down-regulated $(P<0.05)$, and the expression of Bax protein was significantly upregulated $(P<0.05)$ compared to the control group. A comparison between the myocardial model and SP groups revealed that $\mathrm{Bcl}-2$, Beclin-1, $\mathrm{LC} \otimes / \otimes$ protein expressions were significantly down-regulated $(\mathrm{P}<$ $0.05)$ but Bax protein expression was significantly up-regulated $(P<0.05)$ in the myocardial model group.

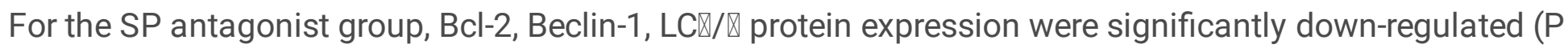
$<0.05)$ while Bax protein expression was significantly up-regulated $(P<0.05)$ compared to the SP group.

\section{The effect of SP on the pathological changes of the myocardium in rats with doxorubicin}

Based on Figure 5, the cardiomyocytes of the control group were neatly arranged without any inflammatory factor infiltration, the myocardial cell structure of the myocardial model group was disordered with myocardial hypertrophy and a large number of inflammatory factors infiltrated, the cardiomyocytes of the SP group were more neatly arranged and had fewer inflammatory cells, while the cardiomyocytes of the SP antagonist group were arranged disorderly and a large number of inflammatory cells that infiltrated.

\section{The effect of SP on cardiomyocyte apoptosis induced by Adriamycin}

According to Figure 6, the apoptosis rate of the myocardial model group was significantly increased compared to the control group $(\mathrm{P}<0.05)$ while the apoptosis rate of the SP group was significantly decreased compared to the myocardial model group $(P<0.05)$ and the SP antagonist group mortality rate was significantly increased compared to the SP group $(P<0.05)$.

\section{The effect of SP on the level of myocardial autophagy in rats with myocardial model induced by Adriamycin}

As observed in Figure 7, the autophagy level of the myocardial model group was significantly reduced compared to the control group, $(P<0.05)$, while the autophagy level of the SP group was significantly increased compared to the myocardial model group $(P<0.05)$ and the autophagy level of the SP antagonist group significantly decreased compared to the SP group $(P<0.05)$.

The effect of SP on the expressions of Bax, Bcl-2, Beclin-1 and LCD/ $\square$ protein in the myocardial tissue of rats with a doxorubicin-induced myocardial model 


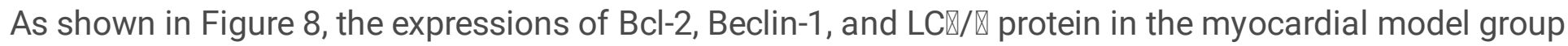
were significantly down-regulated $(P<0.05)$, and the expression of Bax protein was significantly upregulated $(P<0.05)$ compared to the control group. For the myocardial model group compared to the SP

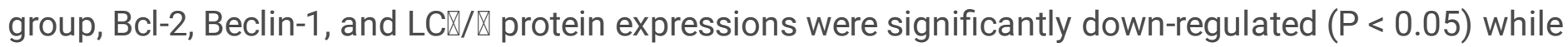
Bax protein expression was significantly up-regulated $(P<0.05)$. The SP antagonist group's Bcl-2, Beclin$1, L C \otimes / \otimes$ protein expression were significantly down-regulated $(P<0.05)$, and Bax protein expression was significantly up-regulated $(\mathrm{P} \otimes 0.05)$ compared to the SP group.

\section{Discussion And Conclusion}

In summary, doxorubicin-induced $\mathrm{H} 9 \mathrm{C} 2$ myocardial cell viability decreased and apoptosis rate increased, and rats with doxorubicin-induced heart failure had an increased myocardial apoptosis rate and decreased autophagy. After SP intervention, the viability of $\mathrm{H} 9 \mathrm{C} 2$ cardiomyocytes induced by doxorubicin was significantly increased. The rate of apoptosis was reduced, and the rate of myocardial apoptosis in rats with heart failure decreased, with an increase in autophagy level. This process could be related to the

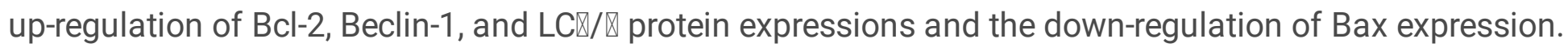

\section{Abbreviations}

Substance P: SP

Neurokinin A: NKA

Neurokinin B: NKB

Eppendorf tube: EP tube

Phosphate-buffered saline: PBS

Sodium dodecylbenzene sulfonate gel electrophoresis: SDS-PAGE

Chemiluminescence: ECL

Dulbecco's Modified Eagle Medium: DMEM

Ethylenediaminetetraacetic acid: EDTA

\section{Declarations}

Ethics approval and consent to participate: All applicable international, national, and/or institutional guidelines for the care and use of animals were followed.

12 adult male SD rats, weight about 220g (manufacturer: Hunan Slack Jingda Experimental Animal Co., Ltd., license number: SCXK (xiang) 2019-0004) 
The study was submitted to and approved by the Key Research and Development Programme of Jiangxi Province ethics committee.

*Key Research and Development Programme of Jiangxi Province. Award number: S2019ZPYFB1176.

* Anesthesia and execution: The animals were sacrificed using lethal injections. rats in each group were executed by intraperitoneal injection of $1 \%$ pentobarbital at a dose of $150 \mathrm{mg} / \mathrm{kg}$.

The study ethics approval was provided by IACUC (Institutional Animal Care and Use Committee).

We have followed (The Guide) for the standard care of animals.

Consent for publication: A written consent for publication was obtained from the hospital experiment committee.

Availability of data and material: All data can be found at the Jiangxi provincial people's hospital.

Competing interest: None declared.

Funding: Key Research and Development Programme of Jiangxi Province (CN) financially supported the whole research. All thr research fees were covered by the 'Key Research and Development Programme of Jiangxi Province (CN)'.

\section{Authors' contributions:}

MYI wrote the manuscript.

$\mathrm{HJ}$ collected all the relevant materials including the figures and medical records.

XMB performed the experiments.

All authors have read and approved the manuscript.

Acknowledgement: Extended thanks to Jiangxi provincial people's hospital research department who helped and encouraged us to complete this research.

Code availability: The code can be found at the Jiangxi provincial people's hospital.

Each of the authors confirms that this manuscript has not been previously published and is not currently under consideration by any other journal. Additionally, all of the authors have approved the contents of this paper and have agreed to the policies of the journal.

\section{References}


1. Biala A.K., Kirshenbaum L.A. The interplay between cell death signaling pathways in the heart. Trends Cardiovasc. Med. 2014;24:325-331. doi: 10.1016/j.tcm.2014.08.002. [PubMed] [CrossRef] [Google Scholar].

2. Qi D., Fu M. Cardiomyocyte apoptosis in heart development: Methods and protocols. Methods Mol. Biol. 2012;843:191-197. [PubMed] [Google Scholar].

3. Pennefather JN, Lecci A, Candenas ML, et al. Tachykinins and tachykinin receptors: a growing family. Life Sciences. 2004;74:1445-63. [PubMed] [Google Scholar].

4. Ustinova EE, Bergren D, Schultz HD. Neuropeptide depletion impairs postischemic recovery of the isolated rat heart: role of substance P. Cardiovasc Res. 1995;30:55-63. [PubMed] [Google Scholar].

5. D'Souza M, Garza MA, Xie M, et al. Substance P is associated with heart enlargement and apoptosis in murine dilated cardiomyopathy induced by Taenia crassiceps infection. J Parasitol. 2007;93:1121-7. [PubMed] [Google Scholar].

6. Kemp M G. Crosstalk Between Apoptosis and Autophagy: Environmental Genotoxins, Infection, and Innate Immunity[J]. Journal of Cell Death, 2017, 9(2): 33-39.

7. Radogna F, Dicato M, Diederich M. Cancer-type-specific crosstalk between autophagy, necroptosis and apoptosis as a pharmacological target[J]. Biochemical Pharmacology, 2015, 94(1):1-11.

8. Oral O, Akkoc Y, Bayraktar O, et al. Physiological and pathological significance of the molecular cross-talk between autophagy and apoptosis[J]. Histology \& Histopathology, 2016, 31(5):479-488.

9. Nikoletopoulou V, Markaki M, Palikaras K, et al. Crosstalk between apoptosis, necrosis and autophagy[J]. Biochimica Et Biophysica Acta, 2013, 1833(12):3448-34 .

10. Wang X F, Ge T T, Fan J. The role of substance P in epilepsy and seizure disorders[J]. Oncotarget, 2017, 8(44):78225-78233.

11. Nikoletopoulou V, Markaki M, Palikaras K, et al. Crosstalk between apoptosis, necrosis and autophagy[J]. Biochimica Et Biophysica Acta, 2013, 1833(12):3448-34 .

12. Levick $S P$, Meléndez G C. Targeting substance $P$ and relaxin: A future combination therapy approach for heart failure?[J]. International Journal of Cardiology, 2016, 204:154-155.

13. Levick S P, Meléndez G C. Targeting substance P and relaxin: A future combination therapy approach for heart failure?[J]. International Journal of Cardiology, 2016, 204:154-155.

14. Valdemarsson $S$, Edvinsson L, Ekman R, et al. Increased plasma level of substance $P$ in patients with severe congestive heart failure treated with ACE inhibitors[J]. Journal of Internal Medicine, 2010, 230(4):325-331.

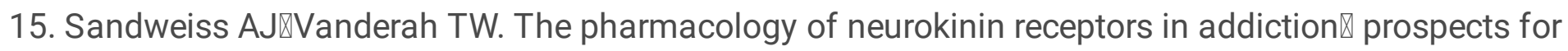
therapy[J]. Subst Abuse Rehabil, 2015, 6(2): 93-102》

16. Wang L, Guo L L, Wang L H, et al. Oxidative stress and substance P mediate psychological stressinduced autophagy and delay of hair growth in mice[J]. Archives of Dermatological Research, 2015, 307(2):171-181. 
17. Tsai $\mathrm{WH}, \mathrm{Wu} \mathrm{CH}, \mathrm{Yu} \mathrm{H}$ J, et al. I -Theanine inhibits proinflammatory PKC/ERK/ICAM-1/IL-33 signaling, apoptosis, and autophagy formation in substance P-induced hyperactive bladder in rats[J]. Neurourology \& Urodynamics, 2016, 36(2):297- 07.

\section{Figures}

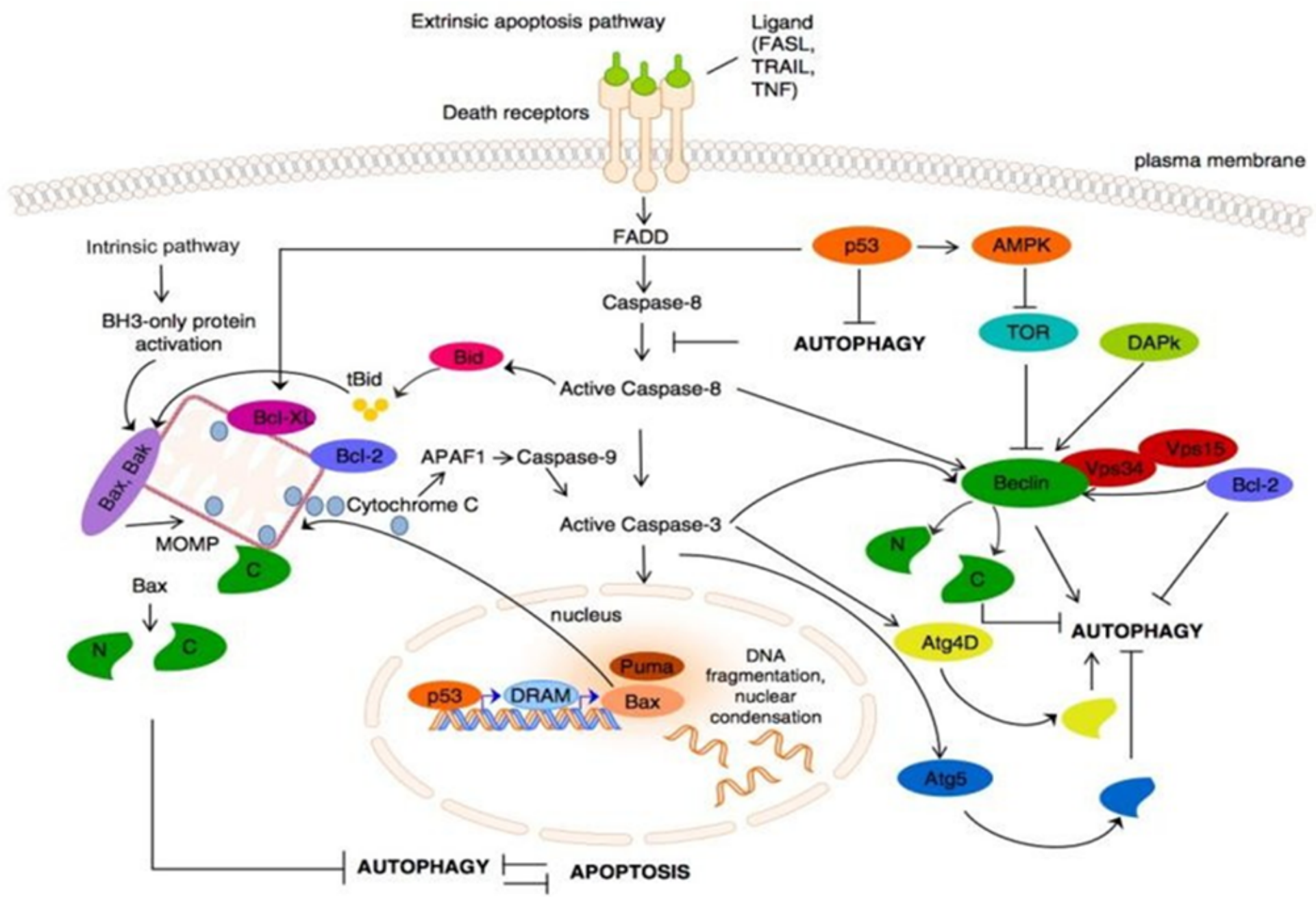

Figure 1

Schematic diagram of the signal pathway of autophagy and apoptosis crosstalk [11] 


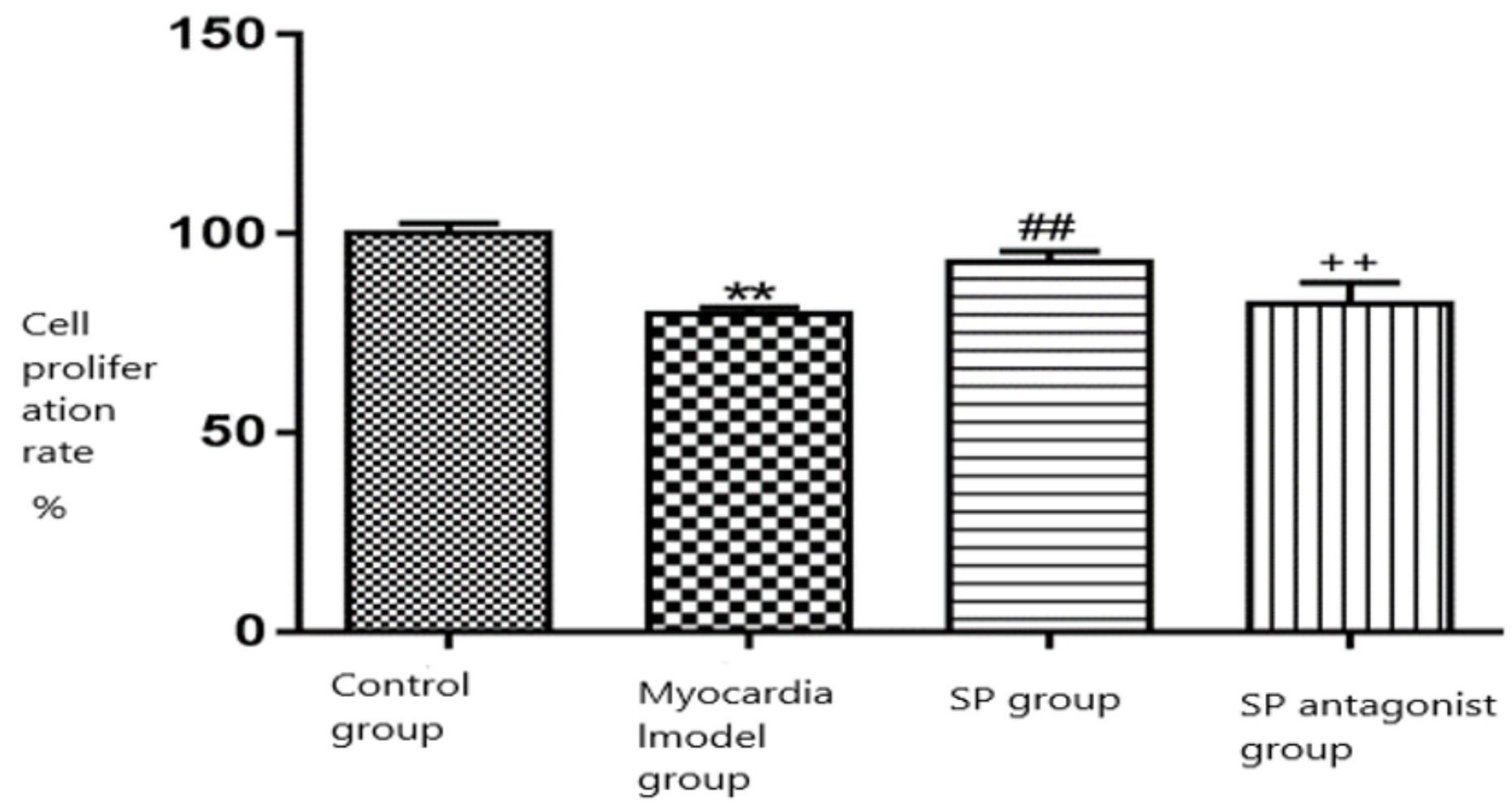

Figure 2

The effect of SP on the viability of $\mathrm{H} 9 \mathrm{C} 2$ cells induced by doxorubicin. Compared to the control group, ** $\mathrm{P}<0.05$; compared to the myocardial model group, \#\# $\mathrm{P}<0.05$; compared to the SP group, $++\mathrm{P}<0.05$ 

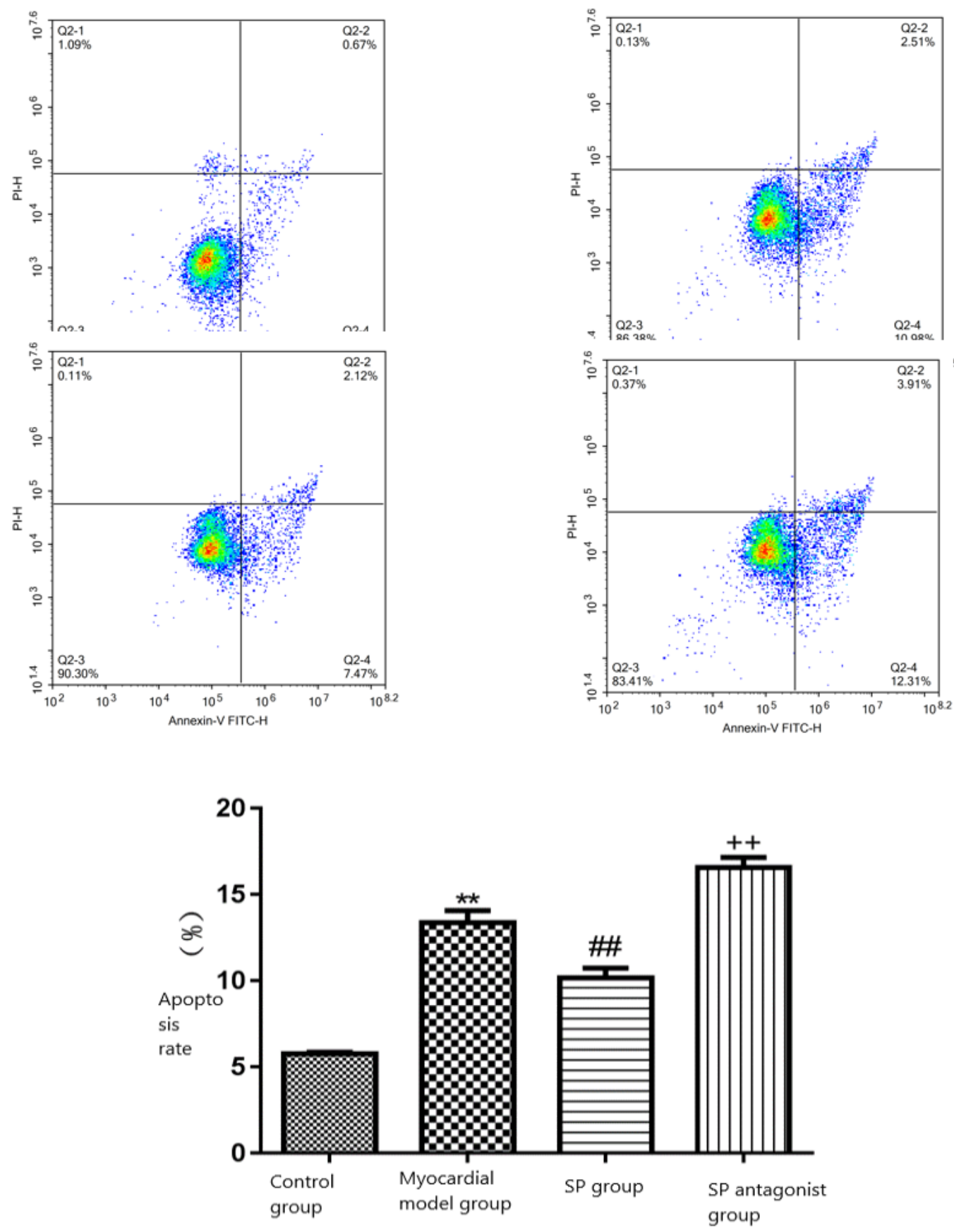

Figure 3

Effect of SP on $\mathrm{H} 9 \mathrm{C} 2$ cell apoptosis induced by Adriamycin. Compared to the control group, $* * \mathrm{P}<0.05$; compared to the myocardial model group, \#\# P $<0.05$; compared to the SP group, $++\mathrm{P}<0.05$ 


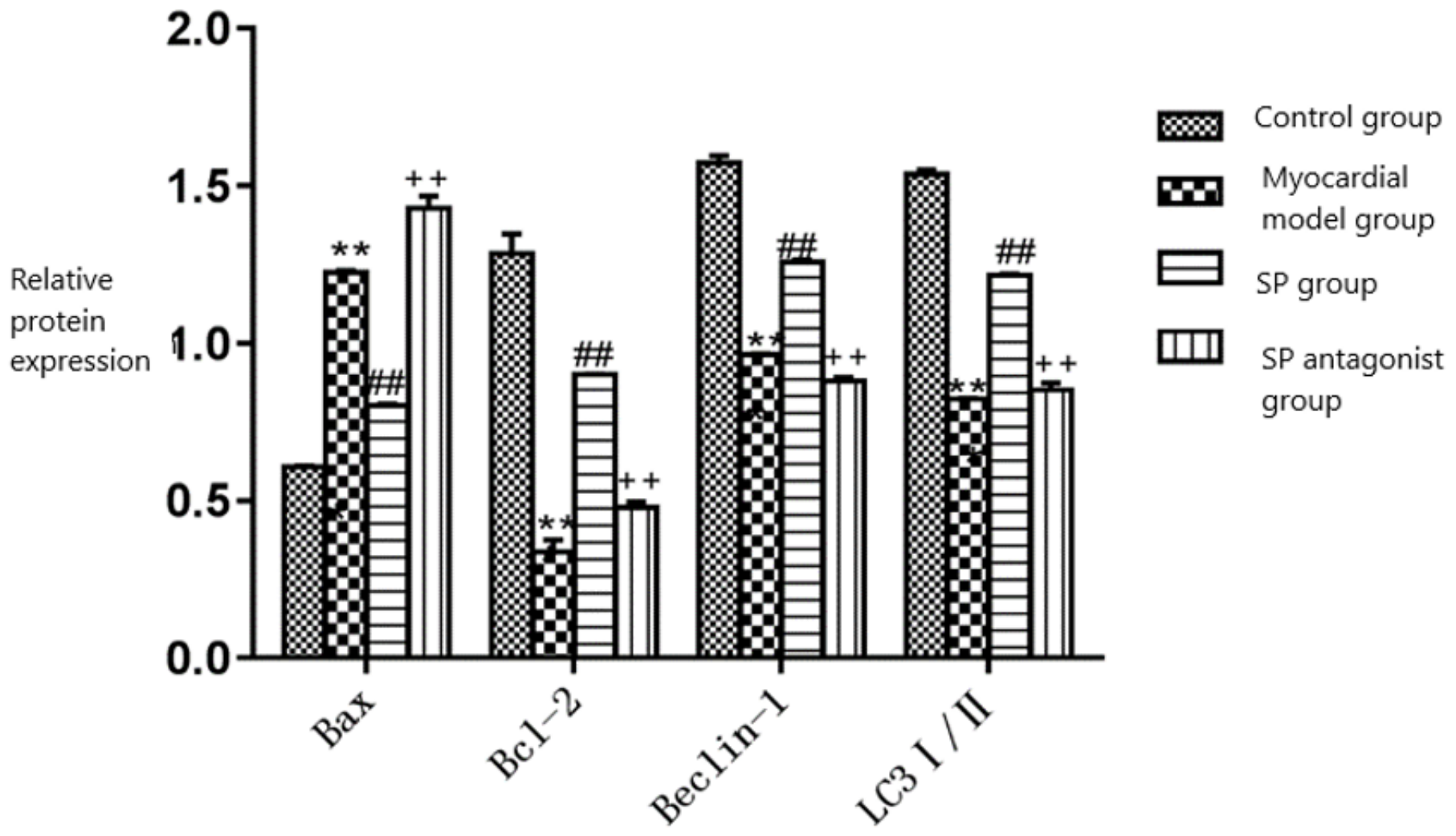

Figure 4

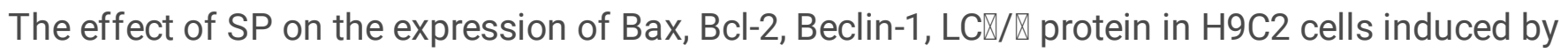
doxorubicin. Compared to the control group, $* \star \mathrm{P}<0.05$; compared to the myocardial model group, \#\# $\mathrm{P}<$ 0.05 ; compared to the SP group, $++\mathrm{P}<0.05$ 


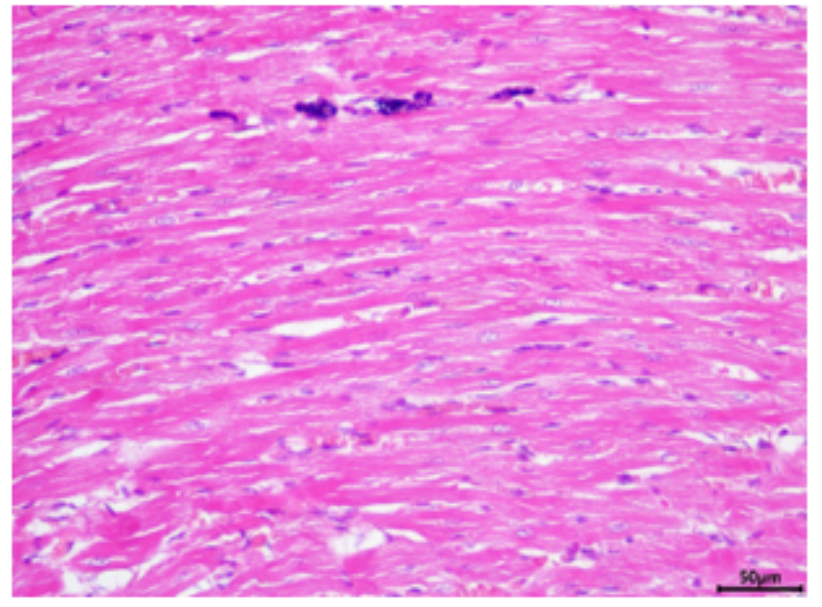

Control

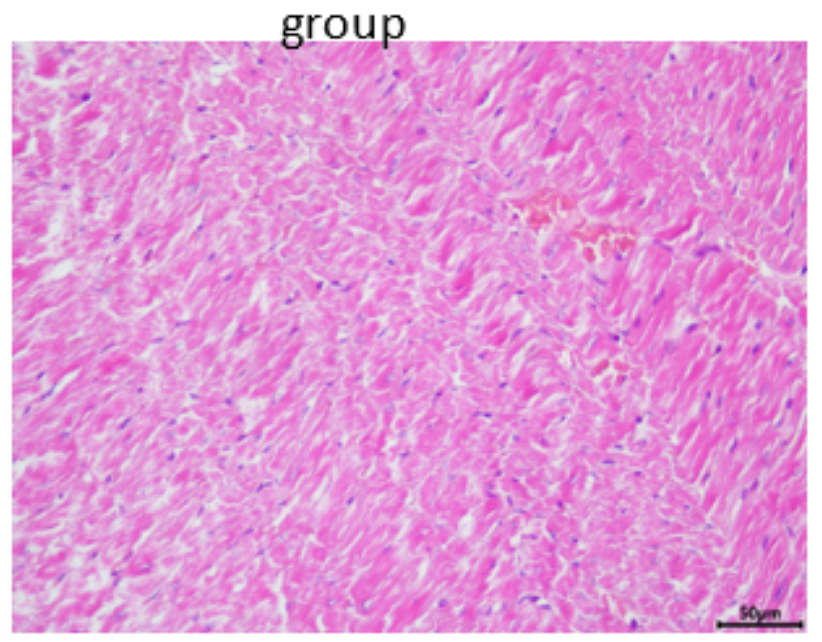

SP

group

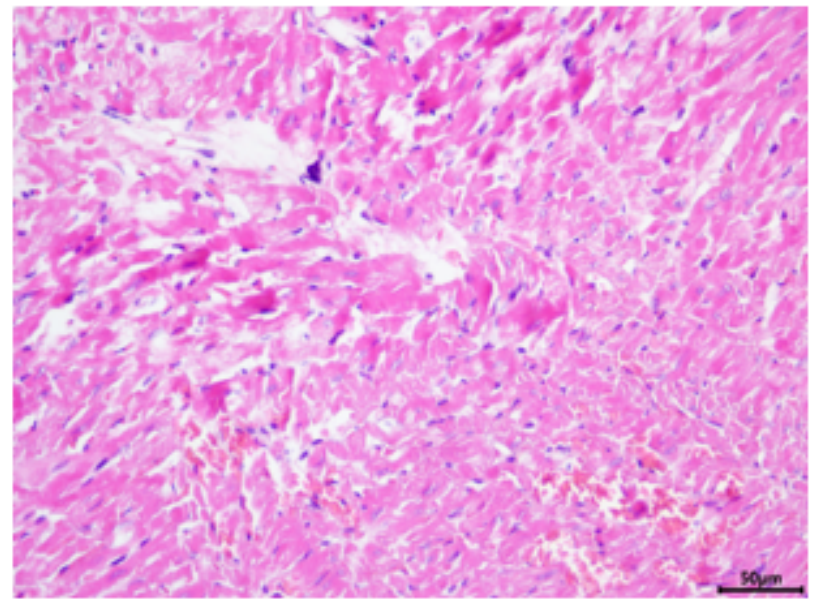

Myocardial model group

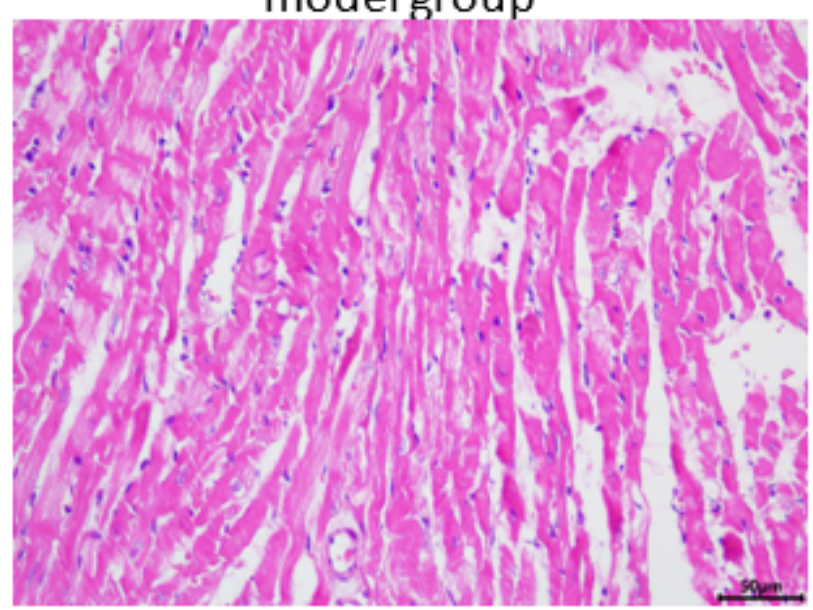

SP

antagonist

group

Figure 5

The effect of SP on the pathological changes of myocardium in rats with myocardial model induced by Adriamycin $(\times 200)$. 

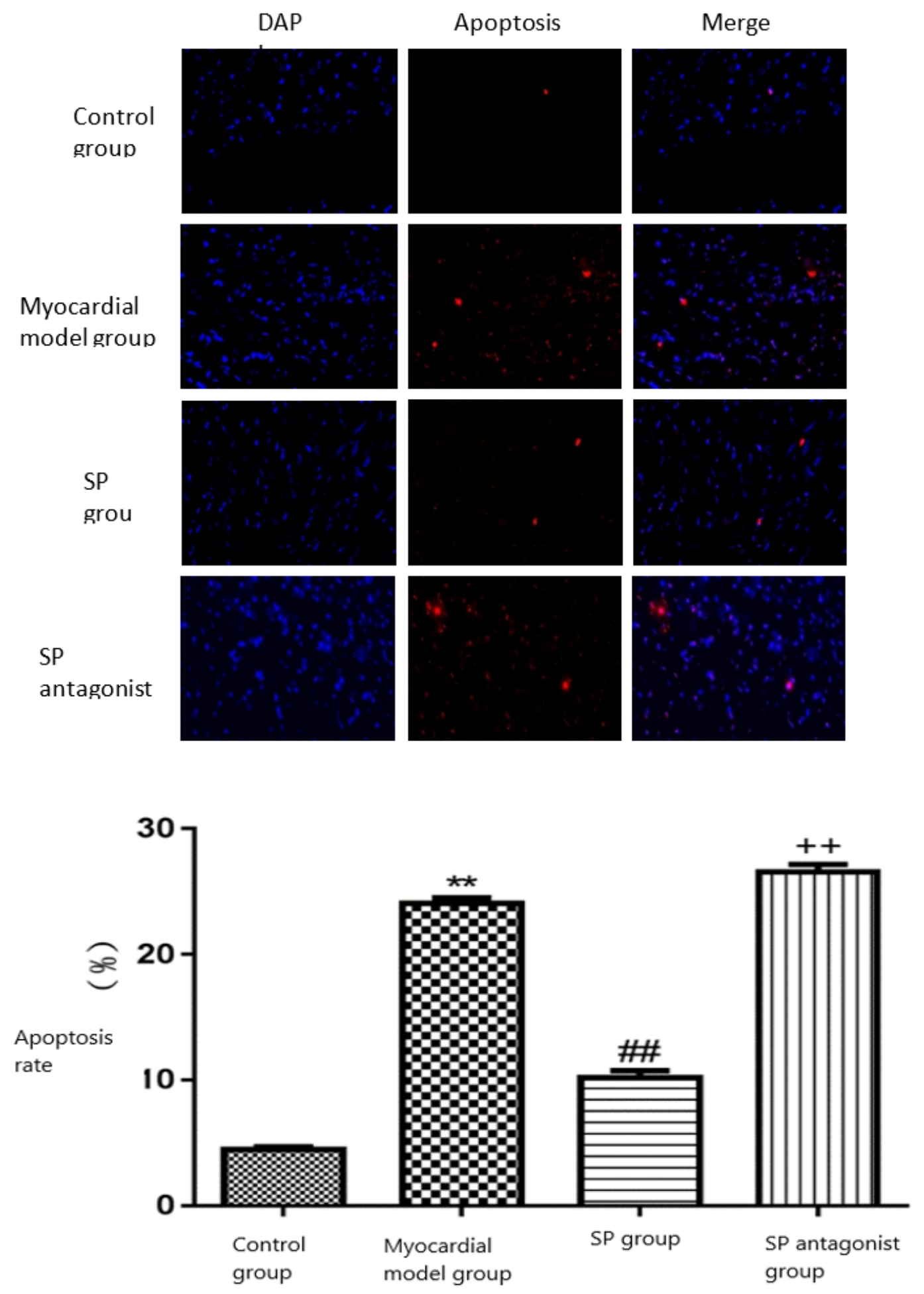

Figure 6

The effect of SP on doxorubicin-induced cardiomyocyte apoptosis in myocardial model rats. Compared to the control group, $* * \mathrm{P}<0.05$; compared to the myocardial model group, \#\# $\mathrm{P}<0.05$; compared to the SP group, $++\mathrm{P}<0.05$ 


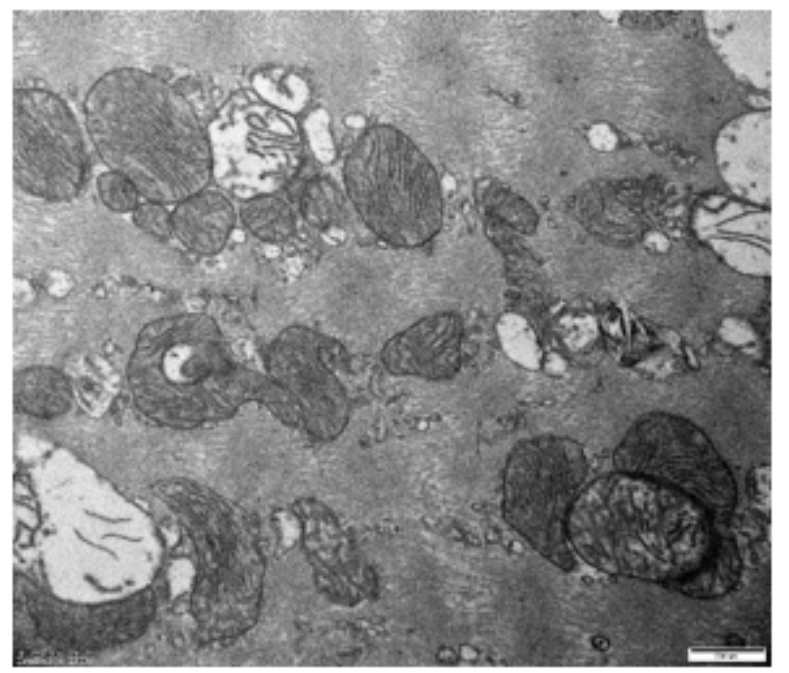

Control

groun

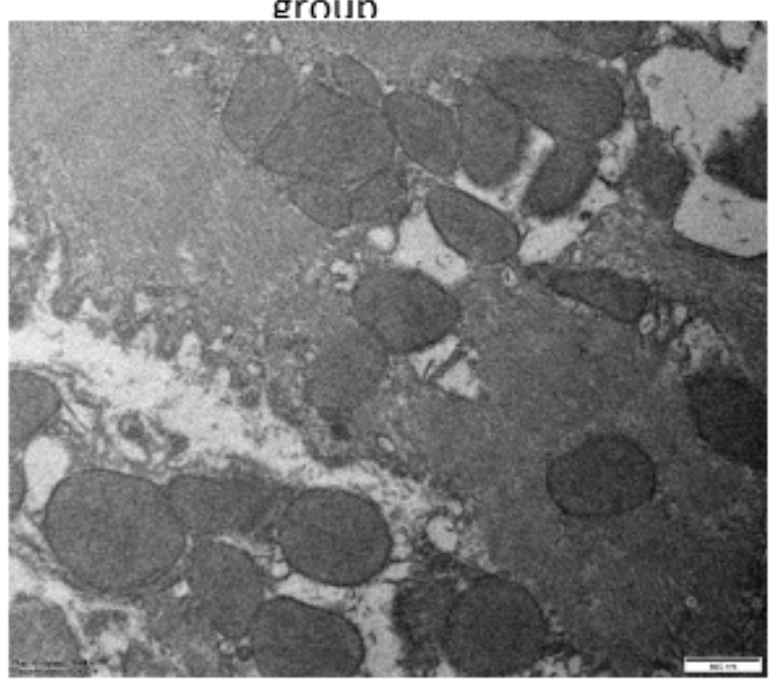

SP

group

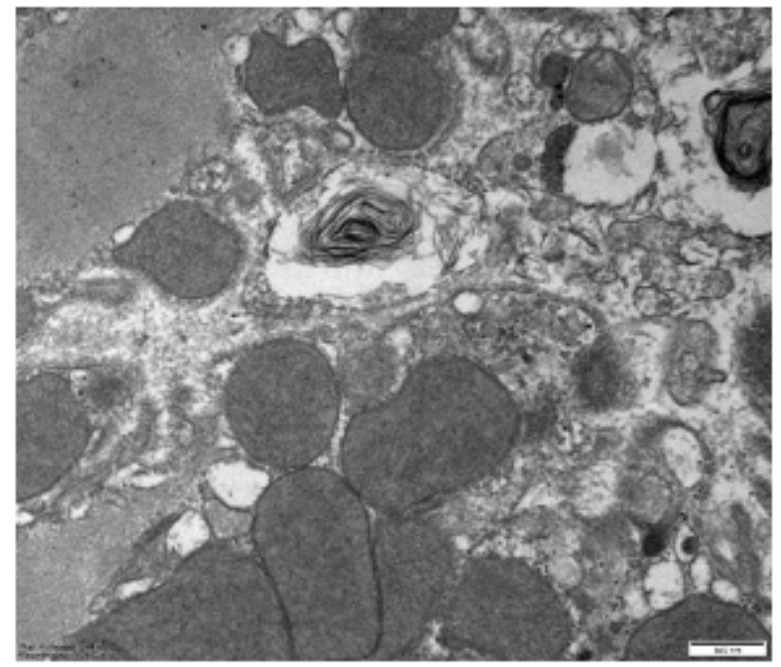

Myocardial model

group

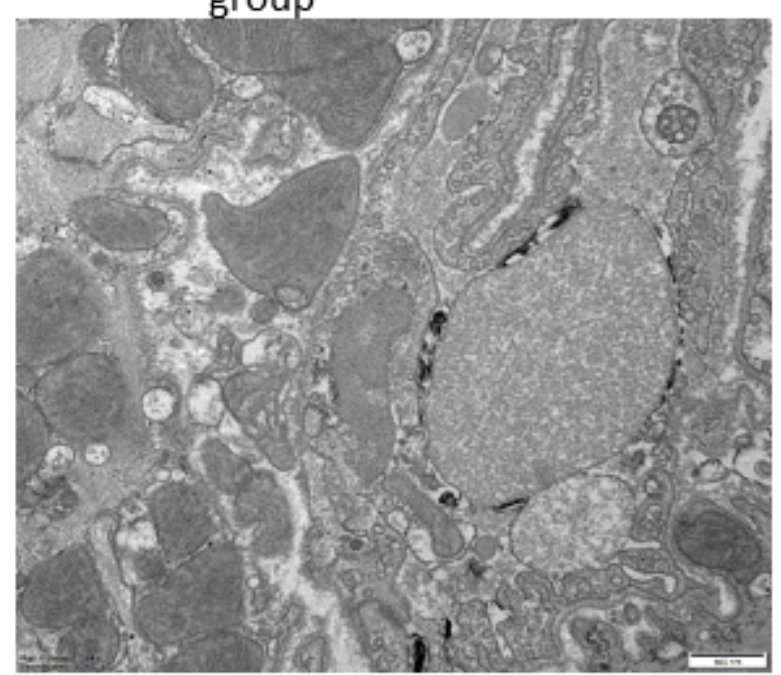

SP antagonist group

Figure 7

The effect of SP on the level of myocardial autophagy in rats with doxorubicin-induced myocardial model. Compared to the control group, ${ }^{*} \mathrm{P}<0.05$; compared to the myocardial model group, \#\# $\mathrm{P}<0.05$; compared to the SP group, $++\mathrm{P}<0.05$ 


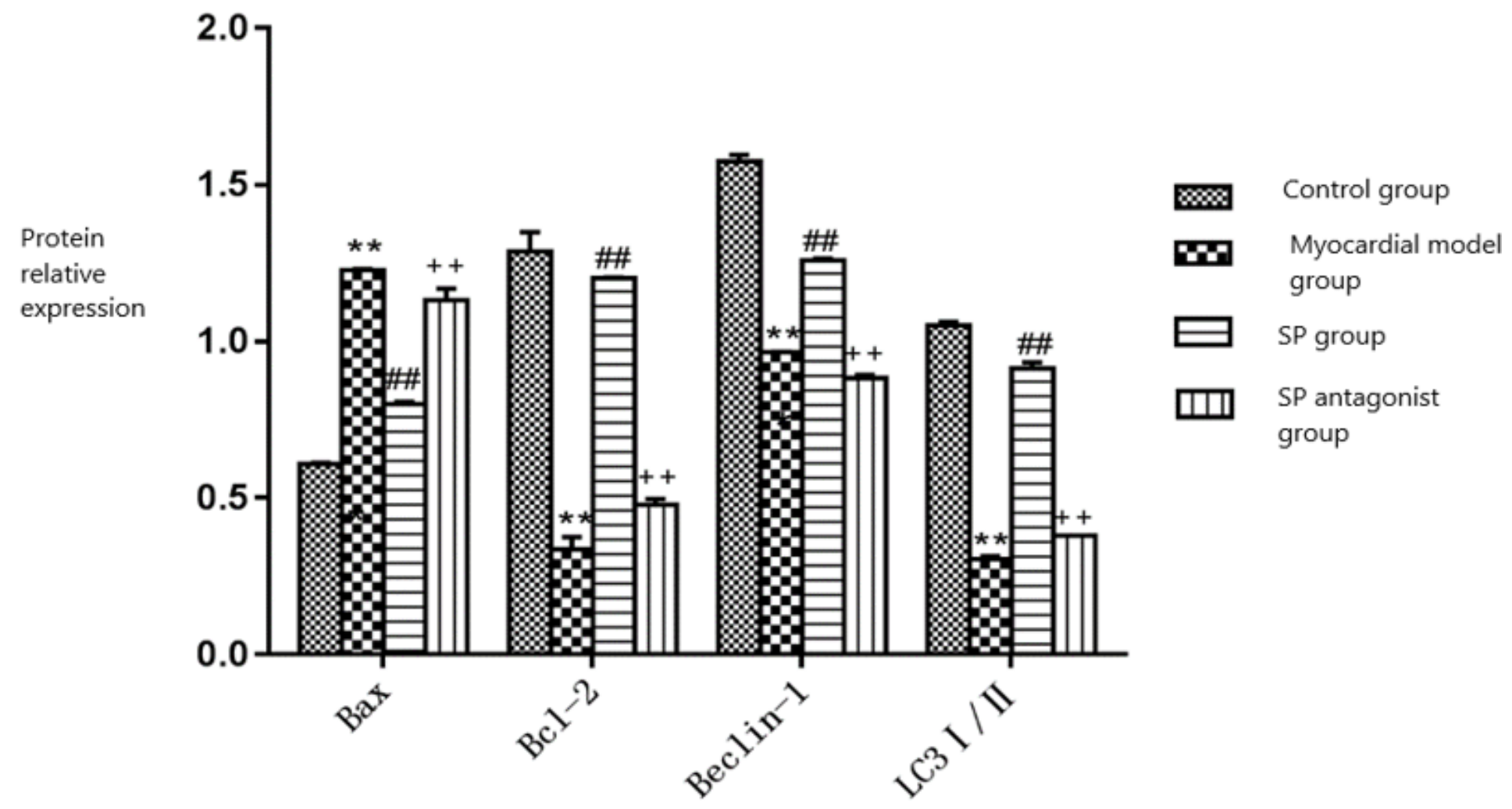

Figure 8

The effect of SP on the expression of Bax, Bcl-2, Beclin-1, LCQ/ $/$ protein in the myocardial tissue of the doxorubicin-induced myocardial model rat. Compared to the control group, $* \star P<0.05$; compared to the myocardial model group, \#\# $\mathrm{P}<0.05$; compared to the SP group, $++\mathrm{P}<0.05$

\section{Supplementary Files}

This is a list of supplementary files associated with this preprint. Click to download.

- AuthorChecklistFull.pdf 